\title{
EDIT: An Educational Design Intelligence Tool for Supporting Design Decisions
}

\author{
Alaa Bafail ${ }^{1}$, Jonathan Tepper ${ }^{1}$, Ann Liggett $^{2}$, Fahd Banakhr $^{3}$ \\ School of Science and Technology, Nottingham Trent University, UK ${ }^{1}$, \\ School of Social Sciences, Nottingham Trent University, $U K^{2}$, \\ Instrumentation and Control Engineering, Yanbu Industrial College, KSA ${ }^{3}$
}

\begin{abstract}
The use of models, frameworks, and toolkits in learning design serve to support teaching practitioners in producing well-structured learning designs for students. However, existing learning design tools inherently lack an objective metric system which is able to measure the degree to which an educational design is well-formed according to either the principles of constructive alignment or more generally design practices that students find satisfactory in practice. Such a metric system, that could integrate measures of educational theory and practice, would enable teaching practitioners to make more informed design decisions such as which profile of activities/assessments to use for a particular set of learning outcomes. This paper presents the first computational intelligence tool that measures educational design quality in a way that is underpinned by both the theoretical principles of constructive alignment and how it is used in practice. Furthermore, the alignment metrics computed are calibrated by student satisfaction scores to promote those structures that are preferred in practice rather than from a theoretical standpoint thus offering more pragmatic and realistic design solutions.
\end{abstract}

\section{Introduction}

Designing for learning is a complex task and considered one of the most fundamental activities of a teaching practitioner [5]. The theory of constructive alignment is considered to be one of the most influential theories in higher education. It is an outcomes-based methodology for designing, promoting, and assessing deep student learning ensuring that all aspects of teaching, from intended learning outcomes, teaching and learning activities, and assessment tasks are all aligned to each other [4]. This guarantees appropriate and therefore effective student learning opportunities. Well-balanced (or constructively aligned) teaching practices have been proven to foster deep student learning [12] and thus impact highly on student satisfaction as clearly documented in [1] and [17].
The design and promotion of constructively aligned teaching practices has been supported to some degree by the development of software tools that attempt to support the teaching practitioner in the design process and assist them in the development of conscious and purposeful teaching. With the creation of different toolkits in this domain, tools differ significantly in terms of how they are structured and the types of pedagogical patterns provided to aid the design process. However, current learning design tools do not either encode a quantitative measure on which to base alignment or any means in which to adapt design patterns according to the student experience.

In this paper we therefore present the first implementation of Tepper's alignment metric module [20] which represents and computes the level to which an educational design is constructively aligned. The tool is able to provide 'alignment metrics' for both holistic and individual aspects of a module design. We then present our approach for an adaptive intelligent learning design system that utilises artificial neural networks [18] to calibrate alignment metrics within the system according to effective practices as judged by student satisfaction. We refer to this resulting computationally intelligent learning design system as the Educational Design Intelligence Tool (EDIT). The purpose of EDIT is to support teaching practitioners in revising design decisions to better promote both the alignment of the system to the intended learning outcomes whilst restricting such decisions to those which engender deep student engagement and satisfaction (even if it violates a number of the underlying alignment principles). Finally, we reveal a number of design preferences formed by EDIT.

\section{Literature Review}

Constructive alignment is an outcome-based design methodology for optimizing the conditions for quality learning, through its integration of instructional design and constructivist principles. It helps teaching practitioners to design, promote, and 
assess deep student learning [4]. Its fundamental assumptions are constructivist rather than instructivist where students constructs their own learning through relevant learning activities and teachers create a learning environment that supports learning activities appropriate to achieving the desired outcomes. This involves selecting the most appropriate teaching and learning activities and assessment tasks for each of the learning outcomes. Usually this selection is governed by the main verb in the outcome statements or objectives and refers to the specific components activities that lead the students towards the outcomes [4]. Figure 1 below illustrates the main components of a module design and how these components inter-relate in a systemic way.

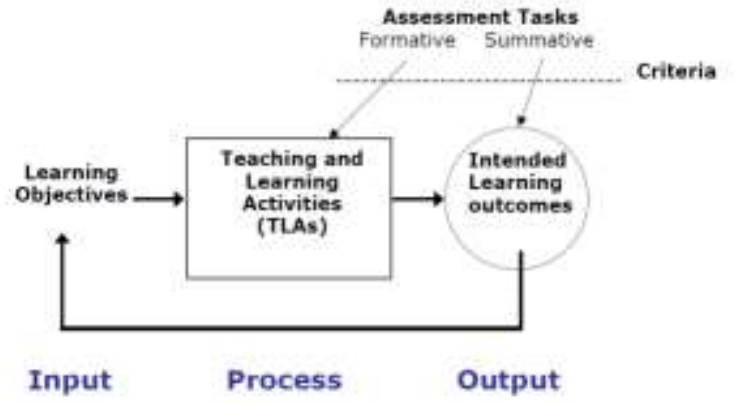

Figure 1. Components of a module design and their interrelationship

The design and promotion of constructively aligned teaching practices has been supported to some degree by the development of software tools that attempt to support the teaching practitioner in the design process and assist them in the development of conscious and purposeful teaching. Some tools are based on particular pedagogic models or philosophies such the Learning Design Support Environment (LDSE) [10] which is underpinned by an ontological model based on Laurillard's Conversational Framework (CF) [10]. The CF embodies a number of pedagogical theories and understanding of lecturers' design practices as elicited Laurillard and her team through interviews with teaching practitioners. Other tools, such as LAMS [7] and Phoebe [16] integrate instructivist, constructivist and problem-based learning theories to structure patterns to guide the teaching practitioners through the design process and support them in making informative design decisions. Importantly, these tools advise and guide teaching practitioners through the pedagogic design process. This guidance is manifested in several ways. For example, LAMS has an associated activity planner tool that provides a set of structured templates which include advice on using, completing, and repurposing teaching, learning and assessment activities for different educational contexts [6]. Both [3] and [6] commended Phoebe for the quality of its guidance as it incorporates a separate wiki-based online resource of tips, guidance, and digital tools to support the decision-making process. The London Pedagogy Planner (LPP) tool [9] in contrast, embeds the support and guidance within the design and decisionmaking process. It takes the user through a series of design decisions using an inspectable and editable model with specific pedagogical approaches to map between teaching components (e.g. intended outcomes and teaching activities). The LDSE incorporated pedagogical patterns that support sequencing the teaching and learning activities (TLAs) and their timing as a core feature. The design pattern is then interpreted in terms of the TLAs and their properties suggesting alternative activities inferred from comparisons between the user's design decisions and the developed ontological model [13].

Despite the potential of these existing tools, there are several limitations. Firstly, they inherently lack objective metric systems that are able to measure the degree to which an educational design is well-formed according to underlying theoretical principles. Secondly, their use of a static knowledge-based system for guiding the design process i.e. its decision logic is hard-wired in. Therefore, these tools cannot adapt to changing practices or be tolerant of variations that govern the system yet may actually be successful in practice according to students' experiences. This is despite the role of student evaluation of their experience being considered essential elements for enhancing the student learning experience [11], [17]. It can help identify the effective aspects of good designs that promote deep student learning whilst minimizing the use of those that do not [1], [17], and [19]. Integrating theory and effective practice based on student satisfaction scores would subsequently enable practitioners to make more informed decisions as to which teaching or assessment activities to use for what learning objective(s) or outcome(s). Subsequently, there is a real need to identify a metric system that enables education design quality to be measured and therefore managed. In maddition, any such management system should be adaptive and ensure its decision making logic is underpinned not only governing pedagogical theories but also on what students consider to work in practice (as evidenced by high student satisfaction scores).

\section{EDIT's Alignment Metric Module}

To address the measurement problem presented in the previous section, EDIT incorporates an alignment metric module to enable the quality of a module design to be numerically measured based on Tepper's quantitative measure of constructive alignment [20]. Tepper's metric uses Bloom's Taxonomy for categorising the main components of 
the teaching system (learning outcomes (LOs), learning objectives (Lobjs), teaching and learning activities (TLAs), and assessment tasks (ATs)) according to the level of cognitive ability they elicit as provided in [4] and some basic principles of generative linguistics to represent the alignment structures. The computational framework utilizes basic set theory and linear algebra to express, represent and compute alignment. For each educational design, the alignment metric is able to generate three different types of tree structure as shown in Figure 2. The alignment is then computed among three main relations (V1, V2, and V3) where $\mathrm{V} 1$, V2, and V3 defined as:

- $\quad$ V1 the relation between LOs and Lobjs

- $\quad \mathrm{V} 2$ the relation Lobjs and TLAs

- $\quad$ V3 the relation between Lobjs and ATs

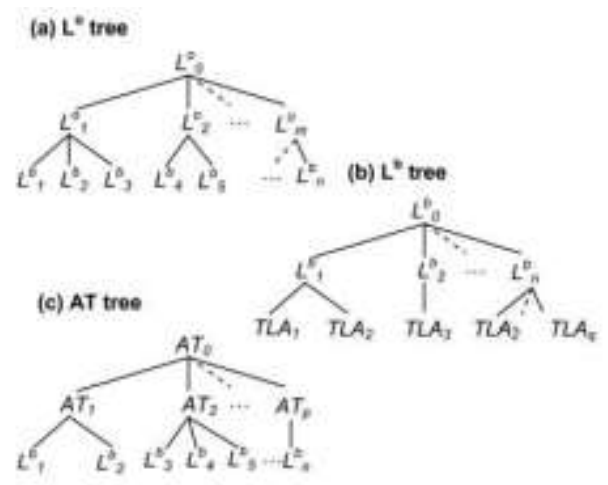

Figure 2. Three different types of tree structure a) showing relationships between outcomes and objectives; b) showing relationships between objectives and TLAs and c) showing relationships between ATs and objectives [20].

The main features associated with EDIT's alignment module are graphically illustrated in Figure 3 and designed to aid teaching practitioners in identifying and relating the core design components symbolically through a graphical user interface whilst abstracting them away from the actual alignment computations used to determine the alignment measures. As input, the module can import existing design patterns from the LDSE, as an XML file, or users can manually input the components of their learning design manually into the system. The module maps the design components to their associated level in Bloom's Taxonomy. The Bloom's Taxonomy levels of the components (based on [4], [20]) are then fed into the alignment metric module to compute and measure how constructively aligned the components are. In order to compute the alignment for a holistic module design, the tool generates three types of tree structures and individually calculates the alignment between learning outcomes and learning objectives, learning objectives and TLAs, and learning outcomes and ATs. The user needs to specify the learning outcomes and the dominated learning objectives in order to generate V1 tree where each learning outcome (parent) can dominate one or more learning objectives (children) likewise for V2 (specify the learning objectives and the dominated TLA(s)) and V3 (specify the learning outcomes and the dominated ATs). V1, V2, and V3 trees need to be balanced via fixing the valence (i.e. the number of children each parent can dominate) for each tree type. If the vectorwidth of a tree is not balanced for a given parent node then filler elements (i.e. <empty_nodes>) are added to make up the number of dominated elements. The empty nodes are set to the level in Bloom's taxonomy indexed by the associated parent node to help maintain equilibrium. The tool then calculates the equilibrium for each relation V1, V2, and V3 via 4-step process as follows: 1) calculates the desired alignment values. The crude assumption made to obtain the desired elements is that given a parent node (e.g. learning outcome) the set of associated children nodes (learning objectives) should elicit the same Bloom's level as the learning outcome (parent node). 2) Calculates the actual alignment values i.e. the the actual level in Bloom's taxonomy referenced by the associated component. 3) Calculates the differences or misalignment (error) values between the desired alignment values and the actual alignment values. Finally 4) calculates the equilibrium alignment value between the parent nodes and the children nodes assigned to them in order to measure the overall alignment between them. This is obtained by calculating the root mean squared error (RMSE) across all elements of the misalignment values between the parent nodes and the children nodes. The overall constructive alignment value for the entire module design is then calculated by aggregating all three relations. According to the computational framework [20], if the parent node refers to a higher level in Bloom's Taxonomy than any child node then 'negative misalignment' is said to occur as the child verbs/activities will not enable the level of the parent to be met. Conversely, if one or more child nodes refer to a higher level of Bloom's Taxonomy than the parent node, then 'positive misalignment' is said to occur as the child verbs/activities will enable students to exceed the level of the parent node. Finally, if the child nodes are exactly the same as the parent node then 'perfect alignment' is said to occur.

The alignment metric module calculates how well aligned the module components are when linked together via interpreting the module design in terms of the core components mentioned above and evaluating the relationship between components in terms of whether they are aligned, negatively misaligned, or positively misaligned. Accordingly, it modifies the misaligned components by suggesting 
alternative and better-suited selections based on principles of constructive alignment. This enables teaching practitioners to adapt their practice to better align their modules by making them aware of misalignments within their educational designs.

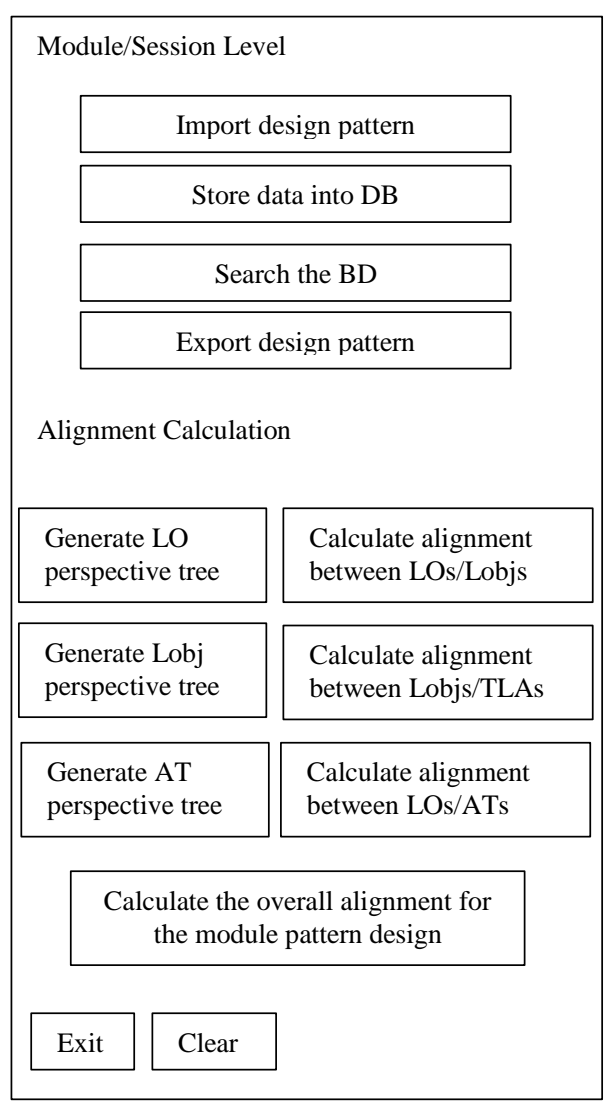

Figure 3. Main features of the EDIT's alignment metric module

The developed alignment tool has been evaluated on a number of module design patterns to measure the degree of alignment and to constructively align the misalignment components. Figure 4 presents an example of four selected module designs with different learning outcomes and the other associated components. The examples are selected randomly and for the purpose of demonstration. The alignment is computed between the different components and the overall module alignment is then given under each module to show the degree of alignment or misalignment each module presents. It is noticeable that there is no balance between the components leading to degrees of negative misalignment in some modules like module 2, 3, and 4. These negative misalignments are indicators of poor design due to first, learning outcomes are not linked with an appropriate Blooms level of learning objectives. Second the TLAs used in these modules are not designed to generate or elicit the desire verb of the associated leaning objectives. Finally, it can be seen that there is little association matching taking place between the learning outcomes and the assessment tasks in module 1 and 2 . The tool attempts to represent the alignment/misalignment numerically with a brief description of the relationship between the components. In order for the modules to reach its equilibrium some modifications are needed.

Therefore EDIT's alignment metric recommended alternative Lobjs, TLAs, and ATs to enhance the relationships between the components according to principle of constructive alignment to elicit the same or higher cognitive level than the associated parent node as shown in Figure 5.

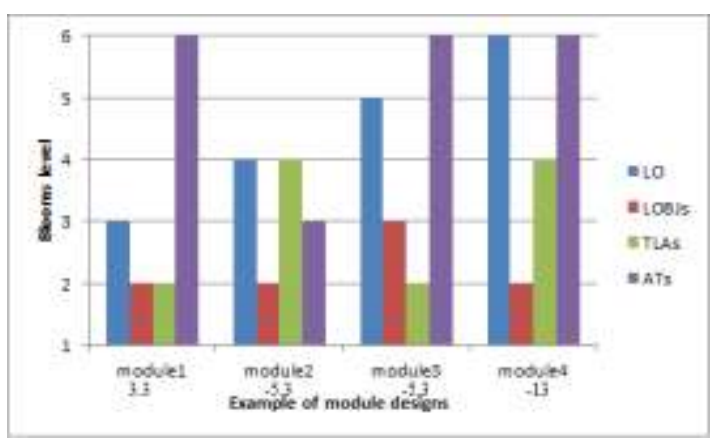

Figure 4. Example of misaligned module designs

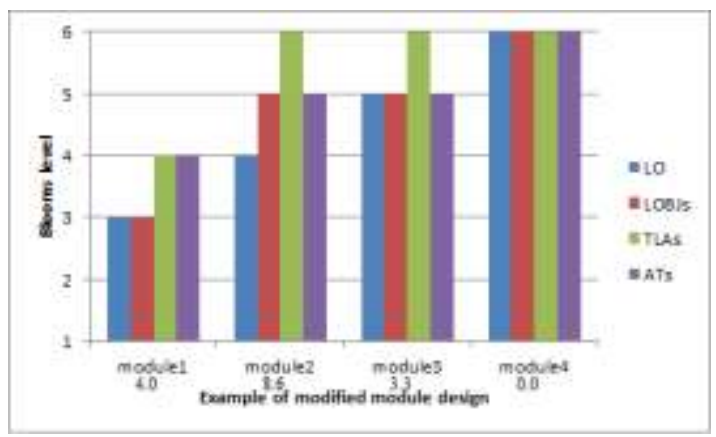

Figure 5. Example of modified module designs

The alignment metric bases both module structure and the categorizations of the different components according to outcomes-based where components are organized according to Bloom's taxonomy and related according to the principles of constructive alignment. This is a top-down theoretical approach to educational design and there is no clear consensus as to what the appropriate alignment values should be in order to determine acceptable design patterns. Subsequently, we have extended the alignment metric module to incorporate and use 'good' design practices, as judged by student satisfaction scores, to calibrate the alignment measures and thus to determine acceptable alignment ranges based on effective practices.

\section{Methodology}

Our methodology consists of the following steps: 
1) Collect learning design patterns with key features such as learning outcomes, learning objectives, TLAs and ATs associated with their levels of student satisfaction scores for the purpose to learn from learning designs of differing quality.

2) Use Tepper's [20] quantitative alignment metric and good design patterns associated with high levels of student satisfaction scores to calibrate alignment measures.

3) Train and optimise a set of artificial auto-encoder neural networks with different configurations and hyper-parameters for memorizing the features of good design patterns (those with high degrees of student satisfaction scores) thus performing an internal memory of the training patterns [2].

4) Select best auto-encoder model based on training performance Root Mean Squared Error (RMSE).

5) Evaluate the selected auto-encoder on test set of poor design patterns to associate patterns with one or more (or combination thereof) the nearest matching 'good' design patterns it has encoded.

6) Identify the design patterns formed from the network and extract the core design preferences discovered from the data.

\section{Calibrating the Alignment Measure by Integrating Theory and Practice}

Effective design practices, as judged by student satisfaction scores, rather than theory in and of itself, was considered as a basis for determining acceptable values for the alignment measure, thus integrating theory and practice into EDIT's decision making. Reviewing good teaching practices that generate high levels of student satisfaction may help to provide useful insight into the value of theoretical alignment values, particularly if there appears to be a strong correlation between the two i.e. alignment scores and levels of student satisfaction. An advantage of this approach will be to help teaching practitioners to better align their module designs in a way that is both theoretically and practically relevant and based on those actually experiencing the impact of the educational design. In order to and use good design practices, design pattern data were extracted from a University Virtual Learning Environment (VLE). In-depth desk based observations covering 567 modules from the University's School of Science and Technology (spanning departments of Physics, Biology, Mathematics, Computing, and Chemistry) were conducted to collect the core educational design components (i.e. LOs, Lobjs, TLAs and ATs) in structural design pattern format. A total of 621 module design patterns were generated from the conducted process. The student satisfaction scores associated with the module design patterns were also captured. All design patterns were then processed by the Alignment Module to calculate and ascribe a constructive alignment score to each core relation (V1, V2, and V3) within the design. In order discriminate the module design patterns based on their effectiveness in practice i.e. by according to the different levels of student satisfaction, the design patterns were rank ordered according to the module's student satisfaction score (via the EvaSys system, a commercial system for creating, distributing and analysing student satisfaction surveys). A five-point Likert scale is used to gauge student satisfaction where 5 refers to 'definitely agree' and thus excellent satisfaction and 1 to 'definitely disagree' and thus poor satisfaction. The design of the questionnaire is aligned to the National Student Survey [14] and therefore covers the same areas of the student learning experience. An EvaSys threshold score of 4 (refers to 'Agree') was selected and therefore the top $84 \%$ (519) of the module design patterns were designated as 'well-formed' design patterns representing the good and effective module design practices. Thus the patterns were used to identify meaningful alignment value ranges for the three main relations (V1, V2, and V3) for Tepper's metric [20]. Note that we calculate V3 differently to that proposed in Tepper's original model. We calculate V3 as the relationship between learning outcomes and ATs (rather than objectives and ATs) as this is more closely related to practice. Applying the metric to the well-formed' design patterns resulted in the alignment value ranges shown in Table 1 . We therefore expect that if module designs stay within these ranges then the modules will be well-formed and constructively aligned in a way that will potentially yield positive student satisfaction.

Table 1. Acceptable and meaningful alignment value ranges calculated from the good and effective module design practices.

\begin{tabular}{|c|c|c|c|c|}
\hline $\begin{array}{c}\text { Alignment value } \\
\text { ranges }\end{array}$ & MIN & MAX & Mean & SD \\
\hline V1 (LO/LObjs) & 4.8 & 11.1 & 7.91 & 3.21 \\
\hline V2 (LObj/TLAs) & 6.9 & 11.5 & 9.26 & 2.38 \\
\hline V3 (LO/ATs) & 7.8 & 13.1 & 10.45 & 2.74 \\
\hline $\begin{array}{c}\text { Overall module } \\
\text { alignment }\end{array}$ & 7.6 & 10.7 & 9.21 & 1.62 \\
\hline Student satisfaction & 4 & 5 & 4.5 & 0.28 \\
\hline
\end{tabular}

\section{EDIT's \& its Neural Auto-encoder}

In order to endow EDIT with an adaptive knowledge base that can learn alignment information directly from the good module design patterns, we 
use artificial neural networks as a novel approach for adaptively supporting the educational design process in a way that marries constructive alignment with good design practice and therefore incorporate both theoretical and practice-based models of design practice. Figure 6 illustrates an overview of the proposed modular architecture. The conceptual model is represented by the learning design database and includes the relationships between the core module components and associating learning design patterns with their student satisfaction levels. The database also serves the data layer and provides a couple of operations to work with XML files.

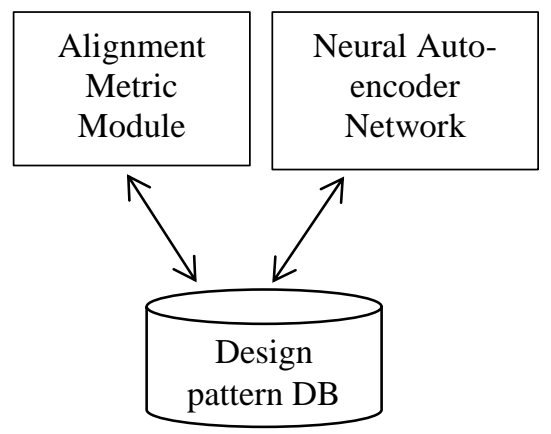

Figure 6. Schematic diagram of EDIT

Artificial neural networks are adaptive systems that have learning properties enabling them to adapt their internal parameters in order to satisfy constraints imposed by a training algorithm and a training set consisting of input and output examples. Instead of following a set of rules specified by a human expert, neural networks are universal function approximators that can learn any function by example [8], [18] and can automatically deduce the underlying rules that describe the input-output relations found in the training set of representative examples. In particular, auto-encoder neural networks (ANN) have been applied to learn the identity function $f(x)=x$ and reconstruct the input data at the output layer with the minimum error construction. The aim of the auto-encoder is to learn a representation (encoding) for a set of data and to output the module design pattern that is on its input layer and thus form a perfect memory of the training patterns. The process of training is called storing the vectors, which can be retrieved from distorted or corrupted input, if the input is sufficiently similar to it [18]. We explored feed-forward multi-layered perceptron networks (FFMLPs) with a broad range of different hidden layer configurations, from shallow to deep networks, as well as different learning algorithms as discussed in more detail in our paper [2]. We used this approach to train our model to specifically memories module design patterns that yield high-levels of student satisfaction (and the related alignment metrics) and therefore to act as a neural auto-encoder. EDIT consists of a neural autoencoder which is trained on 519 design patterns spanning 476 modules from the STEM discipline. As the network is trained to act as a 'perfect memory' of good design patterns, it is anticipated that it will subsequently process test patterns (design patterns of modules with low student satisfaction) as noisy versions of those 'good' design patterns it was trained with, thereby reproducing the nearest 'good' pattern(s) on its output layer and therefore modifying the original design that was presented on its input layer. After successful training, EDIT, was able to learn $88 \%$ of the learning design patterns and generate high test pattern errors thus substantial changes to the input pattern. This infers as the model making suggestions to enhance the module design and thus attract higher level of student satisfaction. Statistical analysis and self-organizing maps have been used to in order to extract and understand the design changes formed by the network. Our preliminary experiments show that in most cases, EDIT was able to correct module designs with poor alignment and student satisfaction in a way that was supported by the pedagogic theory (e.g. using collaborative based and social-constructivist learning activities) and attracted higher levels of student satisfaction. Unlike existing learning design tools, EDIT was therefore able to recommend alternative types of objectives, activities, and assessment tasks relative to the learning outcomes to effect better alignment.

\section{EDIT's Design Preferences}

After evaluating EDIT on the 102 module design patterns found in the test set, we identified that it had formed the following design preferences based on its data-orientated knowledge-base of theory and practice:

1. Both negative and positive misalignment is supported when associating learning outcomes and learning objectives - indicating some confusion in practice as to how these are associated and distinguished.

The V1 (the relation between learning outcomes and dominated learning objectives) alignment scores indicated that the network generated some changes that resulted in lowering the alignment values in most of the cases. This decrease in the degree of alignments has taken two forms where either the learning outcome's level has changed or the learning objective's level has changed. In case where the learning outcomes have changed; the network had made a weak change by moving down one level of the Bloom's level for the following learning outcomes: the analysis (Bloom 4), synthesis (Bloom 5), and evaluation (Bloom 6). This changed to 
application (Bloom 3), analysis (Bloom 4), and, synthesis (Bloom 5) respectively. These changes however, nudged the alignment scores of the test patterns (before) to become within the allowable range. In case where the level of the objective has changed, it was noticed that it has been raised up one level in most cases, which can be seen as a strong change the network has made. This change (moving up one level of Bloom's for the given learning objective) can some time cause the alignment value to be positively misaligned in that learning objectives are eliciting higher Bloom's level than the associated learning outcome level. On the other hand, it was also noticed that the network has decreased the Bloom's level for some learning objectives (moving down one level of Bloom's for the given learning objective) resulting in negative misalignment between the learning outcomes and the learning objectives. As can be seen both both negative and positive misalignment is supported when associating learning outcomes and objectives. This shows that the model is relatively ambivalent towards the alignment between the two components suggesting there is some confusion between teaching practitioners as to how these are related. This is likely because most teaching practitioners find the distinction between learning outcomes and learning objectives somehow nebulous, therefore find it difficult to relate the two.

2. TLAs and ATs that develop or assess higher levels of knowledge and understanding than their associated learning outcomes or objectives are promoted (allowing positive misalignment to occur).

3. TLAs with higher degrees of questioning, interaction and group-based activity are preferred even if this results in significant positive misalignment.

EDIT was typically able to generate strong changes to the V2 (the relation between learning objectives and dominated teaching activities) alignment scores by moving the level of the TLA from lower Blooms level to a higher level represented in activities such as group-based activities, questioning, and interaction thus encouraging collaborative and interactive learning and therefore increasing the level of the corresponding student satisfaction.

4. Essay examinations are preferred over traditional unseen examinations for improving alignment and student satisfaction.

The changes applied to V3 (relation between learning outcomes and dominated assessment tasks) alignment scores has revealed a modification to the alignment theory by using assessment tasks higher than learning outcomes. It also revealed the use of essay type examinations as alternative assessment strategy which can be more effective tool than the traditional unseen exams for improving alignment and student satisfaction. The literatures appear to support this as essay type examinations require less memory and gives a better (compared to non-essay examinations) evaluation of how students have understood the subject and their ability to apply their knowledge and understanding [15].

The implementation and delivery of the design changes applied by the auto-encoder network to each of the V1, V2, and V3 as seen above indicate that there is a relationship between the $\mathrm{V} 1, \mathrm{~V} 2$, and $\mathrm{V} 3$ in relation to student satisfaction whereas the V2 alignment scores $(r=0.645, p<0.05)$ was relatively highly correlated in comparison with V1 and V3 alignment scores $(r=0.504, p<0.05)(r=0.415, p<$ $0.05)$. This correlation was confirmed with calculating the coefficient of determination (0.417) indicating that about $42 \%$ of the total variation in satisfaction is derived from the $\mathrm{V} 2$ that is the relation between learning objectives and the type of TLAs used. The choice of appropriate and effective teaching methods helps to improve the learning of the students by creating interest in the subject and the enthusiasm to learn and developing the creativity sense in the students within themselves. Highly motivated students also tend to be more satisfied with their education [19] and this is achieved by using more teaching methods that engage the students with their learning. Previous studies show that students' academic success and satisfaction relies on certain features of the learning environments, particularly on high level activities such a group work activities and problem-solving exercises [11], [17]. This is quite consistent with the correlation result of $\mathrm{V} 2$ that suggests that whenever there is increasing in the alignment score of V2 by using high-level activities, the student satisfaction score will increase as well.

\section{Conclusion}

We reviewed and discussed the limitations of some existing learning design tools that have been developed in recent years to guide and support teaching practitioners in the learning design. We subsequently introduced, EDIT, a neurally-inspired tool for measuring educational design quality by integrating theory and effective practice to enable practitioners to make more informed learning design decisions. EDIT is an adaptive system so as practice evolves so too can EDIT's underlying knowledgebase by retraining the auto-encoder network. It is able to measure educational design quality and provide meaningful measures for different structures within a design, integrate theory and practice, and learn and adapt to changing practices thus it is a promising tool that addresses the gap identified in 
our introduction. Moreover, in Tepper's alignment model there was no clear consensus as to what the threshold alignment values should be for V1, V2 and V3. In this paper, we have used effective practice (as judged by students) to calibrate these metrics and have thus identified suitable alignment ranges for each of the three tree structures, which researchers and practitioners can utilize and extend.

\section{Future Work}

EDIT represents a data-orientated view of design practices and is dependent on the veracity of its input data. Further research will therefore focus on generating larger samples of module designs that reflect practices across broader subject disciplines and higher education institutions. This will involve the use of focus groups that will help to better establish practices surrounding the use of learning outcomes and learning objectives as there appears much confusion in this area. We will also continue to investigate EDIT's design preferences and how its transformations actually work in practice i.e. do the projected improvements in student satisfaction actually materialize. And finally, to explore how to optimise the deep auto-encoder networks to fully learn the problem and so that they are able to act as perfect memories of the good design patterns (i.e. improve on the current level of $88 \%$ ).

\section{References}

[1] Arbaugh, J. B. "System, scholar, or students? Which most influences online MBA course effectiveness?", Journal of Computer Assisted Learning, 30(4), 349-362, 2014.

[2] Bafail, A., Tepper, J. A and Liggett, A. "Calibrating Educational Design Quality by Integrating Theory and Practice Using Neural Auto-encoders", In Proceedings of ALT Annual Conference 2017: Beyond islands of innovation - how Learning Technology became the new norm(al), 5 - 7 September 2017, University of Liverpool, $\mathrm{UK}, 2017$.

[3] Beetham, H. and Sharpe, R. "Rethinking Pedagogy for the Digital Age. Designing and delivering e-learning". Routledge: London, 2007.

[4] Biggs, J. "Teaching for quality learning at university". Buckingham: SRHE and Open University Press,1999.

[5] Cameron, L. "Could pedagogical planners be a useful learning design tool for university lecturers?" International conference on information communications technologies in education, Rhodes, Greece, 2011.

[6] Conole, G. "Designing for learning in an open world". New York, NY: Springer, 2013.

[7] Dalziel, J. "Implementing Learning Design: The Learning Activity Management System (LAMS)",
Proceedings of the 20th Annual Conference of the Australasian Society for Computers in Learning in Tertiary Education, 2003.

[8] Hornik, K. “Approximation Capabilities of Multilayer Feedforward Networks", Neural Networks, 4(2), 251-257, 1991.

[9] LPP "London Pedagogical Planner". [Online]: http://www.jisc.ac.uk/publications/reports/2008/llpfinal.as px, 2007. (Access Date: 07 November, 2016).

[10] Laurillard, D., Charlton, P., Craft, B., Dimakopoulos, D., Ljubojevic, D., Magoulas, G., Masterman, E., Pujadas, R., Whitley, E.A. and Whittlestone, K. "A constructionist learning environment for teachers to model learning designs," Journal of Computer Assisted Learning, 29: 1530, 2011.

[11] Li, N., Marsh, V. and Rienties, B. "Modelling and Managing Learner Satisfaction: Use of Learner Feedback to Enhance Blended and Online Learning Experience", Decision Sciences Journal of Innovative Education,14(2), pp.216-242, 2016.

[12] Marton, F. and Saljo, R. "On Qualitative Differences in Learning-I. Outcome and process", British Journal of Educational Psychology 46: 4-11, 1976.

[13] Masterman, E., and Craft, B. "Designing and evaluating representations to model pedagogy", Research in Learning Technology 21, no. 0 doi:10.3402/rlt.v21i0.20205, 2013.

[14] NSS, "Thestudentsurvey.com". The National Student Survey.[Online]:http://www.thestudentsurvey.com/the_nss .html\#.Uo9sJMTIbOM, 2013. (Access Date: 05 September, 2016).

[15] O'Farrell, C. "Enhancing Student Learning through Assessment".[Online]:http://learningandteaching.dit.ie/doc uments/assessment_toolkit_v41f.pdf, 2009. (Access Date: 22 November, 2016).

[16] PHOEBE "Phoebe Pedagogy Project". [Online]: http://www.jisc.org.uk/publications/reports/2008/phoebefi nalreport.aspx, 2006. (Access Date: 09 November, 2016).

[17] Rienties, B, Toetenel, L, and Bryan, "A Scaling up' learning design: impact of learning design activities on LMS behavior and performance". Paper presented at the 5th Learning Analytics Knowledge conference, New York, 2015.

[18] Rumelhart, D., Hinton, E. and Williams R. "Learning representations by back-propagating errors". Nature 323, 533-536, 1986

[19] Solinas, G., Masia, M., Maida, G. and Muresu, E. "What Really Affects Student Satisfaction? An Assessment of Quality through a University-Wide Student Survey". Creative Education.Vol.3, 2012.

[20] Tepper, J. A "Measuring constructive alignment: an alignment metric to guide good practice," in 1st UK Workshop on Constructive Alignment, Higher Education 
International Journal for Infonomics (IJI), Volume 10, Issue 2, June 2017

Academy Information and Computer Sciences (ICS), Subject Centre and Nottingham Trent University, UK, 2006. 